\title{
Direct measurements using on-board diagnostics: study of the dynamics of a vehicle equipped with a DSG automated transmission
}

\author{
Dan Micu*, Iordan Fileru, Marius Toma and Cristian Andreescu \\ Automotive Engineering Department, University POLITEHNICA of Bucharest, Splaiul \\ Independentei, Nr. 313, Romania
}

\begin{abstract}
The paper aims to analyze a series of OBD data from a Volkswagen Passat 2.0 tdi, $103 \mathrm{~kW}$, equipped with an automatic DSG transmission, while traveling in the city. Some parameters are analyzed, with a special emphasis on the relation between the engine speed and vehicle speed, presenting also an example of using the information provided by the OBD to determine the gear ratios. The gear steps and the way they are used while driving the vehicle are identified in the end.
\end{abstract}

\section{Introduction}

The OBD system has been introduced to monitor vehicle pollution control systems. For this purpose, it monitors a multitude of engine parameters and, if necessary, it can provide this information to the user or authorities. Access to this information is done with specialized equipment, but cannot be restricted according to current standards.

The continuous development of electronic control of car systems has determined an increasing of information available via OBD. Although access to these data is now easier than ever, the data retrieved still needs to be processed and interpreted [1].

The paper aims to analyze a series of data from a Volkswagen Passat 2.0 tdi, $103 \mathrm{~kW}$, equipped with an automatic DSG (DCT) transmission, in real urban traffic. Some parameters are analyzed, with a special emphasis on the relation between the engine speed and vehicle speed, defining and explaining their ratio.

The ultimate goal is to determine the gears and their use while driving a vehicle equipped with DSG.

A model able to detect active gear using data measured only by the OBD port is developed and validated over experimental results in [2], where a suitable mix of filtering algorithms and rules has allowed overcoming the problems due to uncertainty and discretization error in OBD velocity data.

Usually the information about the selected gear is available on the OBD. This paper proposes a relatively simple method by which the gear engaged at a given moment can be determined when the information about the selected gear is not available.

${ }^{*}$ Corresponding author: $\underline{\text { dan.micu@upb.ro }}$ 
In addition, the overall transfer ratio for each gear is also determined, thus obtaining important data for design and modelling.

\section{Investigation method}

A Foxwell NT100 system was used to retrieve the information available in the vehicle's computer. This is a data logger that once coupled to the OBD socket records the preset parameter values during vehicle movement. Data of each trip is recorded. The device provides information on the distance and duration of the trip, average and maximum speed, hard accelerations and decelerations, etc.

Beside this, for a specific trip, up to four parameters can be recalled and displayed in a plot with time synchronization. Thus, a series of observations, questions, analysis and evaluations which can conduct to interesting and sometime surprising conclusions are allowed.

Since the parameters values are read every second, this kind of plot offers a lot of information at a glance, but for a more precise analysis, the usage of "zoom in" button is recommended. In this case, an increasing of the scale will permit a better analyzing of the interdependence between the engine speed, vehicle speed and manifold air pressure (MAP) shown in Figure 1.

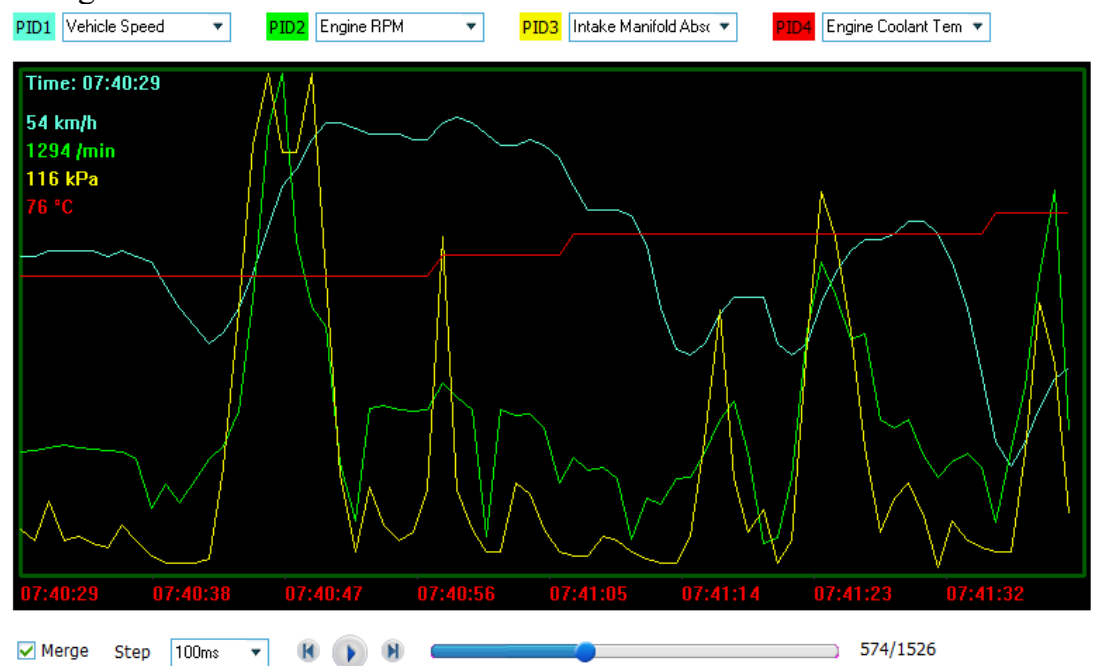

Fig. 1. The evolution of the four parameters merged in the same window on a higher scale.

For a better analysis or to establish a clear relationship between different parameters, the raw data provided by NT 100 can be entered into specialized software (Excel, MATLAB, Mathcad, etc.).

\section{Measuring engine and vehicle speed}

The data shown on the charts offer a multitude of information about the vehicle and the engine. For a better approach, the data are analyzed separately, but not without considering the influences from the other parameters.

To analyze the relation between the engine speed and the vehicle speed, and to determine the gears and their using, a short period from a trip (similar to a driving cycle) was chosen and the data were processed in Excel. 
From evaluation of a recorded trip, it was considered a period of about five minutes, between 7:39:00 and 7:43:57shown in Figure 2. The engine speed values and the vehicle speed values were inserted in Excel and their ratio (n/V) was computed for every second.

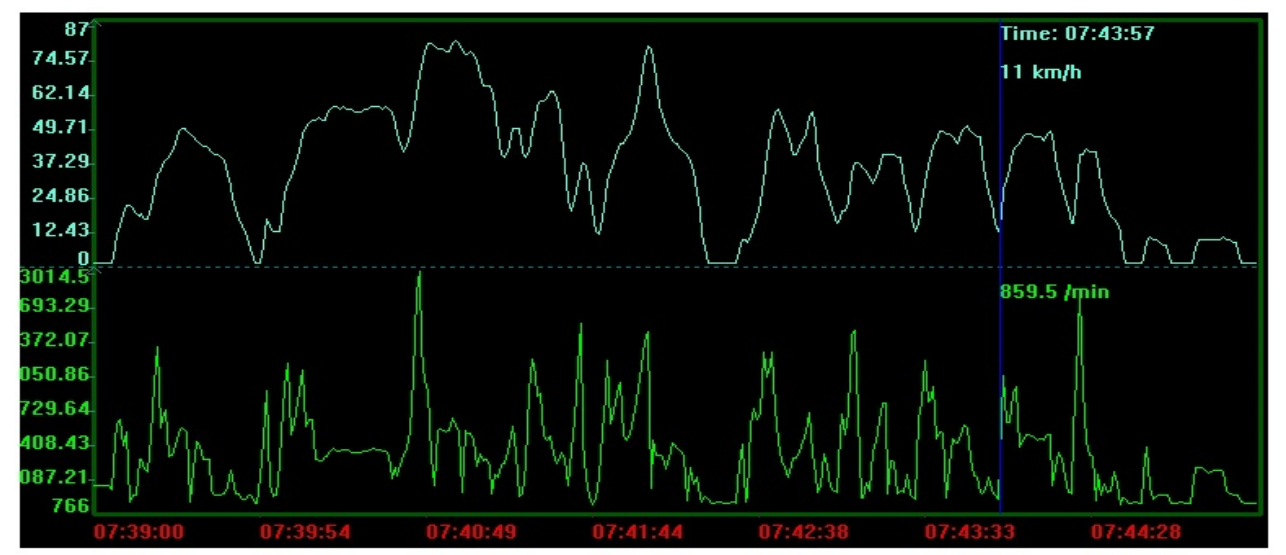

Fig. 2. Vehicle speed and engine speed variation in period selected to be analyzed.

The first step was to arrange data according to the vehicle speed and then to represent all the values of the engine speed corresponding to vehicle speed in the same chart $n(V)$ (Figure 3).

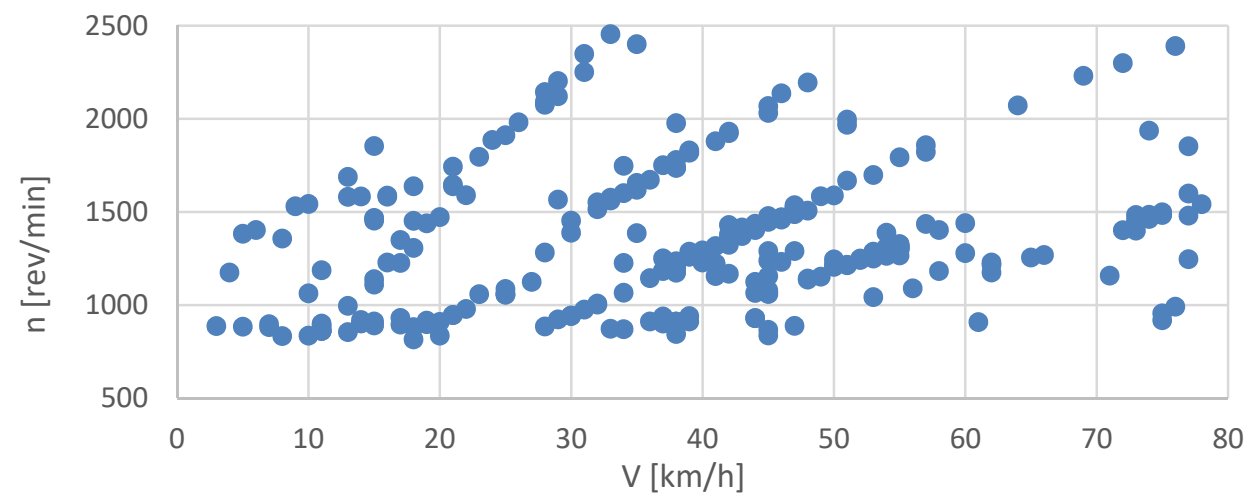

Fig. 3. Distribution of the engine speeds versus vehicle speed $n(V)$.

There is a clear tendency of these values to distribute after some oblique lines, whose slope depends on the transfer ratio of the transmission. Since the slope of these lines is actually the $\mathrm{n} / \mathrm{V}$ ratio, it results that this is not only the transmission ratio but a global ratio between engine speed and vehicle speed, which included the final transmission ratio and rolling radius of tier [3], besides gear ratio.

The unit of measure for this global ratio " $\mathrm{R}$ " will be [rev*h $\left./ \mathrm{min}^{*} \mathrm{~km}\right]$ or, after reduction of time units, the units will become [rev/ $\mathrm{km}]$. This means that if the global ratio "R" will be multiplied by 60 , the result will show how many revolutions of the engine are necessary to travel one $\mathrm{km}$, for a certain gear.

Therefore, the global ratio of the vehicle can be very useful in modeling, because it comprises all the cinematic aspects regarding transmission of the power from engine to road (gear ratio, final ratio, rolling radius) and could be used as a transfer function for a "black box" transmission. 
Determining the slope of the lines direct from the precedent chart is quite difficult. The $(\mathrm{n} / \mathrm{V})$ ratio is calculated and data is sorted by this value to have a predetermination of the global transfer ratios for all the moments of the selected period. Afterward, the dispersion of the ratio values is represented in a chart (Figure 4).

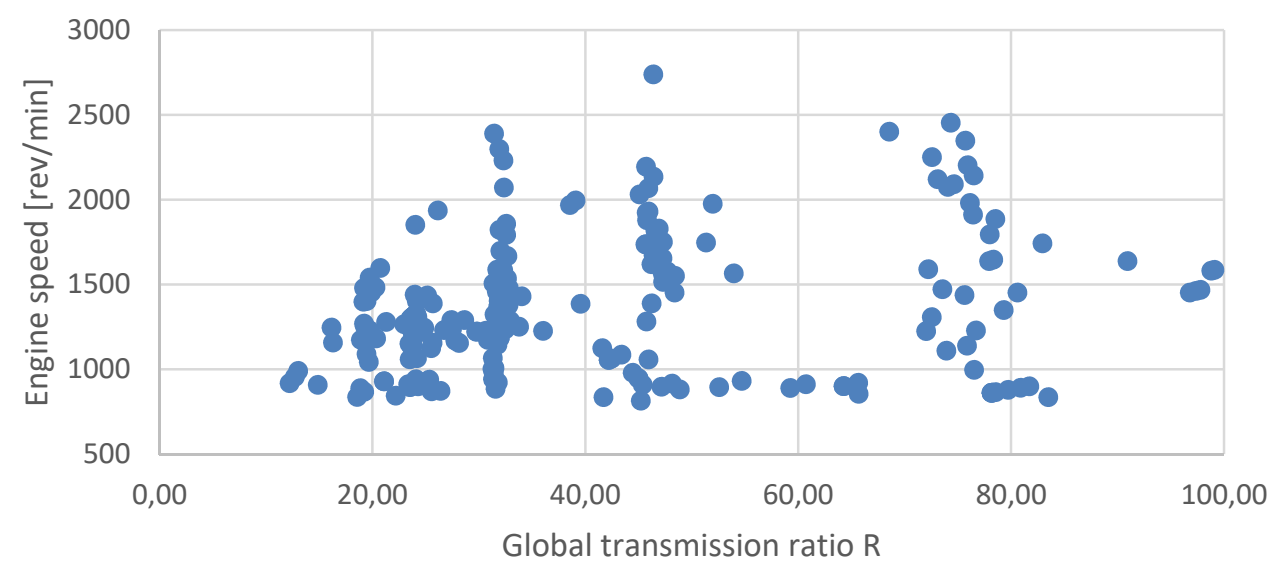

Fig. 4. Dispersion of the global transmission ratio $\mathrm{R}$ values.

\section{Determining the gears and their using}

It can be easily noticed that there are certain concentrations of these points around certain values of the global ratio. In fact, they represent the $\mathrm{R}$ values corresponding to the different gears. For the chart above, these concentration values are about 20, 24, 32, 46 and 75 . The higher the ratio, the lowest the gear.

Apparently, the vehicle seems to be equipped with a 5-speed gearbox. The values above 100 were eliminated, in the previous chart because their dispersion was too high (reaching even 300). Those values would not be used to determine an additional transmission ratio. Otherwise, due to the very large scale, for low values (20, 24 and 32) the concentration of the points could not be distinguished because of the very large scale, for low values $(20,24$ and 32 ).

In fact, the vehicle is equipped with a 6-speed DSG gearbox, which means that first gear is not shown in Figure 6. The first gear has a ratio $\mathrm{R}>100$, somewhere between 120 and 150. It is quite difficult to determine it precisely due to the short time it is used and to the large dispersion, more accentuated than the one that can be observed in the second gear.

This is partly due to the speed reading resolution (as an integer) and partly to the functioning mode of a DSG transmission. In the case of low speeds, reading resolution leads to significant variations of the $\mathrm{n} / \mathrm{V}$ ratio when the vehicle speed value changes with one unit for the same engine speed.

A direct-shift gearbox (German: Direkt-Schalt-Getriebe), commonly abbreviated to DSG, is an electronically controlled dual-clutch multiple-shaft manual gearbox in a transaxle design, without a conventional clutch pedal and with fully automatic or semimanual control [4]. Preselection of the gear that has to be engaged (which in fact implies its coupling) and the change of the gear without interruption of the power flow (which means coupling a clutch simultaneously with the decoupling of the second) are two functional particularities of the DSG. If registration is made by the OBD at the time of gear change, the $\mathrm{n} / \mathrm{V}$ ratio value could be an intermediate value between the ratio values of those two gears. 
To determine the right transmission ratios, these intermediate points are removed and only those whose $\mathrm{n} / \mathrm{v}$ ratios are close to the predetermined $\pm 5 \%$ are selected from all data.

The selected data are sorted by the value of this ratio and they are shown in Figure 5 separately by groups of values so that they can be distinguished. A trend line is assigned to each point group. Its slope (the coefficient of $\mathrm{x}$ ) represents roughly the ratio $\mathrm{R}$. The equation of each straight line appears on the graph along with the average square deviation $\sigma^{2}$.

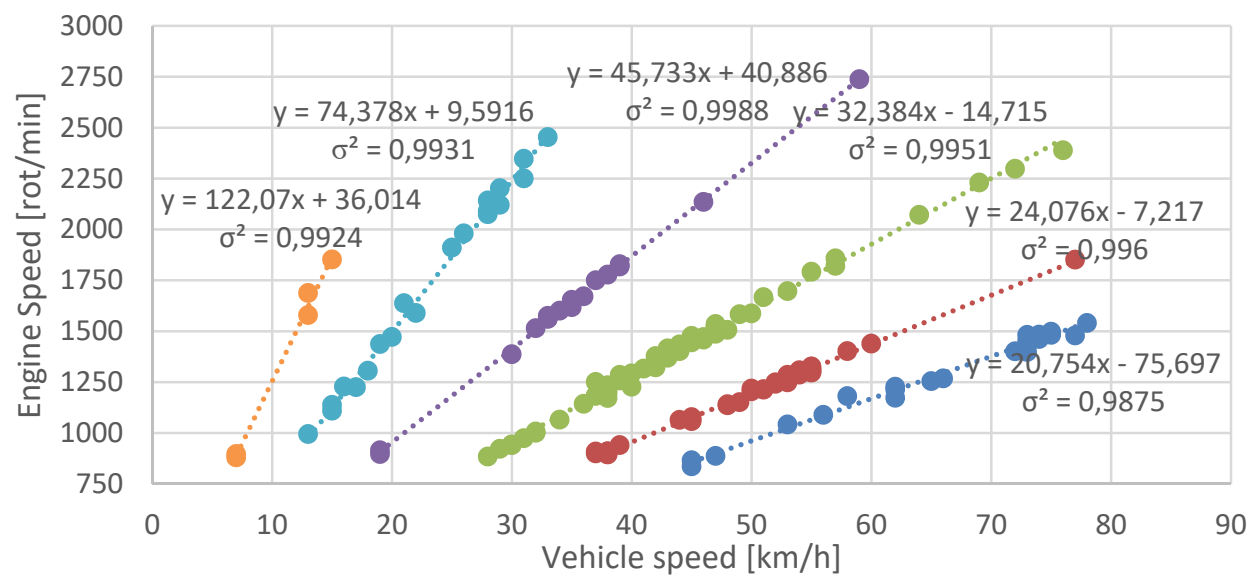

Fig. 5. Correlation between engine speeds and vehicle speed for all gears.

Normally the free term should be 0 (the lines go through the origin), but due to the sources of errors presented above, the points on the graph do not align perfectly and the slope of the straight line does not exactly represent the ratio R. In any case, the average deviation square $\sigma 2$ calculated for each gear is less than 1 . Consequently, the ratio $R$ is determined with sufficient precision to identify the gear but also to be used in modelling.

As previously mentioned, the overall gear ratio $\mathrm{R}$ comprises gear ratio, final transmission ratio and rolling radius. It follows that the dimensions of the tires (which is stored in the car's computer memory) and the final transmission ratio (which can be found on certain profile sites) must be known to compute the correct gear ratio. Values of gear ratios only are from 4.148 for the first gear until 0.686 for the sixth gear [5].Issues of gear shifting optimization still represent actual challenge to scientific-expert potential in the field of motor vehicles [6].

However, the ratio of $\mathrm{R}$ is enough to identify the gears. The corresponding gear is identified for values close to those shown in Figure 5. An intermediate gear which will

Table 1.The gears according to the values of the $\mathrm{R}$ ratio.

\begin{tabular}{ccccccccccc}
\hline R & $\mathbf{1 9 -}$ & $\mathbf{2 2}$ & $\mathbf{2 3 -}$ & $\mathbf{2 8}$ & $\mathbf{3 0 -}$ & $\mathbf{4 0}$ & $\begin{array}{c}\mathbf{4 4 -} \\
\mathbf{4 7}\end{array}$ & $\mathbf{5 2}$ & $\mathbf{6 5}$ & $\mathbf{7 2 - 7 8}$ \\
\hline gear & $\mathbf{6}$ & 5.5 & $\mathbf{5}$ & 4.5 & $\mathbf{4}$ & 3.3 & $\mathbf{3}$ & 2.75 & 2.3 & $\mathbf{2}$ \\
$\mathbf{R}$ & 99 & 107 & $\begin{array}{c}120- \\
130\end{array}$ & 170 & 240 & 300 & $\begin{array}{c}> \\
390\end{array}$ & & & \\
\hline gear & 1.5 & 1.35 & $\mathbf{1}$ & 0.75 & 0.5 & 0.3 & $\begin{array}{c}\mathbf{0} \\
\mathbf{( N )}\end{array}$ & & & \\
\hline
\end{tabular}

Show the gears changes will be considered for intermediate values, according to Table 1 .

Value 0 is considered for the neutral gear. 
All data is reordered according to time after that the gear for each second is known. The engine speed, the vehicle speed and the used gear is graphically shown in Figure 6. For a better graphic representation, the speed is displayed at $1 / 20$ scale.

Given the proximity between the engine speed scale and the $\mathrm{R}$ ratio value for the last gear (6th), the velocity and speed graphs overlap.

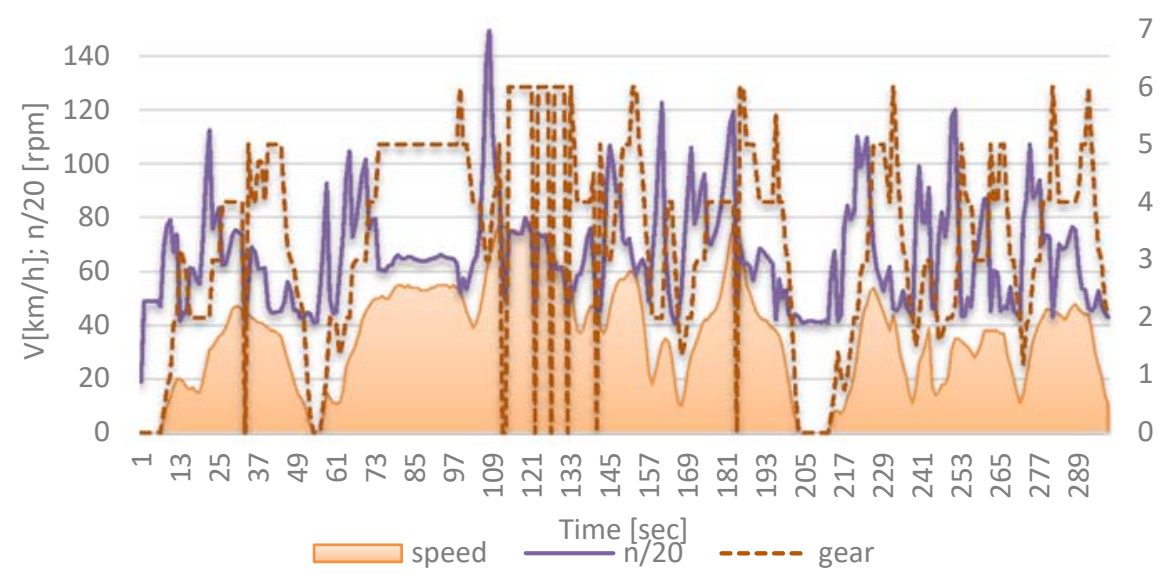

Fig. 6. Engine speed, vehicle speed and their corresponding computed gears.

\section{Conclusions}

The chosen method provides easy access to information of real interest in research and design and deliver fast and cost-effective results for moderate precision determinations.

These data can then be used for modelling. DSG transmission has a fast gear changeover without interruption of power flow that allows gearing to be changed over a period of time without loss of speed.

Changing the gears is done at relatively low engine speeds of 1500-2000 rpm, which leads to the use of the engine very close to the economic pole at partial loads.

Changing the gear to a higher one leads to the use of the engine at a higher torque and a lower engine speed, which in principle leads to a higher load and a closer to the economic pole.

This work has been funded by University Politehnica of Bucharest, through the "Excellence Research Grants” Program, UPB - GEX 2017. Identifier: UPB- GEX2017, Ctr. No. 64 /2017”.

\section{References}

1. W. Colonna,Motor Magazine, June, 18(USA: Hearst Business Publishing, Inc. 2010)

2. M.D'Agostino, M. Naddeo,G. Rizzo, Proc. of the 19th World Cong., IFAC 47, 6618-23 (2014)

3. I.Yadav, Int. Journal of Eng. Dev. and Res., 5, 46-51 (2017)

4. VW of America, The 02E Direct Shift Gearbox Design and Function Self-Study Program Course, (2004)

5. W.Colonna et al., Audi, Jetta, Passat 09G/09M Tech. Service Information (2010)

6. I.A. Blagojevc, G.S.Vorotovc, G.S.Ivanovic, et al. Thermal Science 17, 91-105 (2013) 